\title{
CPTAC Brain Lower Grade Glioma Baseline Form
}

National Cancer Institute

\section{Source}

National Cancer Institute. CPTAC Brain Lower Grade Glioma Baseline Form. NCI

Thesaurus. Code C157531.

Terminology used in support of the data collection efforts of the Clinical Proteomic

Tumor Analysis Consortium (CPTAC) with the focus on Brain Lower Grade Glioma

Baseline Form data. 